Received: 21 June 2017

Accepted: 5 October 2017

Published online: 30 October 2017

\section{Leishmania infantum} lipophosphoglycan induced-
Prostaglandin $\mathrm{E}_{2}$ production in association with PPAR- $\gamma$ expression via activation of Toll like receptors-1 and 2

Jonilson Berlink Lima ${ }^{1,2}$, Théo Araújo-Santos $\mathbb{1}^{1,2}$, Milena Lázaro-Souza ${ }^{1,3}$, Alan Brito Carneiro ${ }^{4}$, Izabela Coimbra Ibraim ${ }^{5}$, Flávio Henrique Jesus-Santos ${ }^{1,3}$, Nívea Farias Luz ${ }^{1}$, Sara de Moura Pontes ${ }^{1,3}$, Petter Franco Entringer ${ }^{6}$, Albert Descoteaux ${ }^{7}$, Patrícia Torres Bozza ${ }^{4}$, Rodrigo Pedro Soares 5 \& Valéria Matos Borges (I) $^{1,3}$

Lipophosphoglycan (LPG) is a key virulence factor expressed on the surfaces of Leishmania promastigotes. Although LPG is known to activate macrophages, the underlying mechanisms resulting in the production of prostaglandin $\mathrm{E}_{2}\left(\mathrm{PGE}_{2}\right)$ via signaling pathways remain unknown. Here, the inflammatory response arising from stimulation by Leishmania infantum LPG and/or its lipid and glycan motifs was evaluated with regard to $P G E_{2}$ induction. Intact $L P G$, but not its glycan and lipid moieties, induced a range of proinflammatory responses, including $\mathrm{PGE}_{2}$ and nitric oxide (NO) release, increased lipid droplet formation, and iNOS and COX2 expression. LPG also induced ERK-1/2 and JNK phosphorylation in macrophages, in addition to the release of PGE 2 , MCP-1, IL-6, TNF- $\alpha$ and IL-12p70, but not IL-10. Pharmacological inhibition of ERK1/2 and PKC affected PGE 2 and cytokine production. Moreover, treatment with rosiglitazone, an agonist of peroxisome proliferator-activated receptor gamma (PPAR- $\gamma$ ), also modulated the release of $\mathrm{PGE}_{2}$ and other proinflammatory mediators. Finally, we determined that LPG-induced PPAR- $\gamma$ signaling occurred via TLR1/2. Taken together, these results reinforce the role played by $L$. infantum-derived LPG in the proinflammatory response seen in Leishmania infection.

Visceral leishmaniasis (VL) is caused by species from the Leishmania donovani complex. In the New World and Europe, this disease is mainly linked to L. infantum, which is widespread throughout Latin America, including Brazil, accounting for approximately $90 \%$ of all VL cases. VL is one of the most severe types of leishmaniasis and can prove lethal if untreated. Several determinants of VL virulence/pathogenicity have been attributed to intraspecies variation among $L$. infantum strains, in addition to host immune response ${ }^{1}$.

${ }^{1}$ Gonçalo Moniz Institut, Oswaldo Cruz Foundation (FIOCRUZ-BA), 40296-710, Salvador, BA, Brazil. ${ }^{2}$ Center of Biological Sciences and Health, Federal University of Western Bahia (UFOB), 47808-021, Barreiras, BA, Brazil. ${ }^{3}$ Federal University of Bahia (UFBA), 40110-170, Salvador, BA, Brazil. ${ }^{4}$ Laboratory of Immunopharmacology, Oswaldo Cruz Institut, FIOCRUZ-RJ, 21040-900, Rio de Janeiro, RJ, Brazil. ${ }^{5}$ René Rachou Institut, Oswaldo Cruz Foundation (FIOCRUZ-MG), 30190-002, Belo Horizonte, MG, Brazil. ${ }^{6}$ Federal University of Rio de Janeiro (UFRJ), NUPEM, Campus Macaé, 27933-378, Macaé, RJ, Brazil. 'Institut National de la Recherche Scientifique, Institut Armand-Frappier, H7V 1B7, Laval, Canada. Jonilson Berlink Lima and Théo Araújo-Santos contributed equally to this work. Rodrigo Pedro Soares and Valéria Matos Borges jointly supervised this work. Correspondence and requests for materials should be addressed to R.P.S. (email: rsoares@cpqrr.fiocruz.br) orV.M.B. (email: vborges@bahia.fiocruz.br) 
Lipophosphoglycan (LPG) is a major Leishmania surface glycoconjugate. This pleiotropic virulence factor is crucial during host-parasite interaction in both vertebrate and invertebrate hosts ${ }^{2}$. At early infection stages, LPG inhibits the complement system, favors opsonization by macrophages, impairs phagolysosome maturation and inhibits protein kinase $\mathrm{C}$ activation $^{3-9}$. LPG is comprised of a glycan core, to which carbohydrate residues are added by different enzymes ${ }^{10}$. Biochemically, LPGs demonstrate intra- and inter-species variability with regard to repeat units. For example, L. infantum exhibits three types of LPG (I, II and III), each with different glucosylation levels, whereas L. donovani and L. braziliensis are devoid of side-chains in their repeat units ${ }^{11-13}$. While these biochemical differences are determinant to a pleiotropic range of immune responses in the vertebrate host, the isolated immunomodulatory properties of intact L. infantum LPG and/or its glycan and lipid moieties remain to be determined.

LPGs of different Leishmania species are potent agonists of Toll-like receptors (TLRs), especially TLR2 and TLR $4^{14-18}$. Early studies revealed that purified LPG from L. major activates TLR2 and induces the nuclear translocation of NF-kB via Myd88 ${ }^{19,20}$. Regarding New World species of Leishmania, purified LPG from L. infantum and L. braziliensis exhibits antagonic properties. LPG from L. braziliensis and L. mexicana are very pro-inflammatory, activating the ERK $1 / 2$, JNK and $\mathrm{p} 38$ pathways via TLR $2^{18,21}$. An interesting feature in the pathway activation profile of L. infantum LPG is its ability to gradually induce JNK and p38 subsequent to MAPK activation, whereas the profile associated with L. brasiliensis LPG is very transient ${ }^{21}$. L. amazonensis LPGs have been reported to induce NO, TNF- $\alpha$ and IL- 6 via TLR4 and but were not capable of translocating NF-kB ${ }^{17}$.

The balance between lipid mediators, mainly the eicosanoids leukotriene $\mathrm{B}_{4}\left(\mathrm{LTB}_{4}\right)$ and prostaglandin $\mathrm{E}_{2}$ $\left(\mathrm{PGE}_{2}\right)$, is also an important component of the inflammatory response to and outcome of infection by intracellular pathogens ${ }^{22}$. Previous in vitro studies have demonstrated the role of $\mathrm{LTB}_{4}$ as a parasite killing mechanism, while $\mathrm{PGE}_{2}$ favors Leishmania survival ${ }^{23-28}$. More recently, lipid mediators have been identified as biomarkers during cutaneous ${ }^{29,30}$ and visceral leishmaniasis ${ }^{31}$. In addition, L. amazonensis or its lipophosphoglycan is known to induce neutrophil activation, degranulation and leukotriene $\mathrm{B}_{4}\left(\mathrm{LTB}_{4}\right)$ production ${ }^{23}$. Nevertheless, the role of LPGs in the macrophage activation of eicosanoid production pathways, such as $\mathrm{PGE}_{2}$, is still unknown.

It has been well established that during inflammatory responses, intracellular organelles known as lipid droplets (LDs) are the main sites where enhanced eicosanoid production takes place, e.g. $\mathrm{PGE}_{2}$ production by cyclooxygenase-2 (COX-2) ${ }^{32}$. The ability of $L$. infantum-infected cells to induce increased LD formation was recently demonstrated ${ }^{33}$. PGE 2 production machinery has been associated with PPAR- $\gamma$ expression. The COX-2 gene promoter presents a PPAR- $\gamma$ response element (PPRE), which indicates that the induction of PPAR $\gamma$ regulates COX-2 expression and, consequently, $\mathrm{PGE}_{2}$ production ${ }^{34}$. In addition, PPAR- $\gamma$ also participates in TLR2-induced LD formation and $\mathrm{PGE}_{2}$ production in macrophages ${ }^{35}$. In this scenario, COX-2 plays an important role as a downstream pathway that is engaged during TLR activation by Leishmania parasites or their PAMPs ${ }^{36}$.

In the present study, we evaluate the role of $L$. infantum LPG and its derived fragments in triggering a proinflammatory immune response related with $\mathrm{PGE}_{2}$ production by macrophages. Only intact LPG, not its derived moieties, was found to trigger the TLR-1/2-induced signaling pathway via PPAR- $\gamma$ activation, thereby contributing to a $\mathrm{PGE}_{2}$-associated inflammatory response.

\section{Results}

Intact Leishmania infantum LPG stimulates macrophage activation in a proinflammatory response associated with $\mathrm{PGE}_{2}$ production.

Differences have been described with regard to intact $L$. infantum LPG and its lipid and glycan moieties derived from BA262 and BH46 strains in terms of the biochemical structure of glycidic side chains ${ }^{13}$ (Supplemental Fig. 1). Here, were tested whether these distinct LPG molecules are capable of inducing differential macrophage activation in vitro. IFN- $\gamma$-primed bone marrow-derived macrophages (BMDM) were incubated with LPG from BA262 or BH46 strains for 24 hours. Intact LPG from the BA262 and BH46 strains, but not their lipid and glycan moieties, induced $\mathrm{PGE}_{2}$ and $\mathrm{NO}$ production (Fig. $1 \mathrm{~A}$ and B). Similarly, an increased number of LDs was observed only in intact LPG-stimulated cells (Fig. 1C). To demonstrate the involvement of LPG in the activation of enzymes implicated in $\mathrm{PGE}_{2}$ and $\mathrm{NO}$ production, protein expression of COX-2 and iNOS was determined by Western Blotting. IFN- $\gamma$-primed BMDMs stimulated for 24 hours with intact LPG, lipid and glycan moieties, exhibited robust iNOS and COX-2 expression only under stimulation by intact LPG (Fig. 1D).

LPG from the BA262 strain was chosen for the further experiments in order to better represent the most common unbranched LPG in L. infantum, since this molecule was well characterized as a type I LPG, found in the majority of strains (90\%), including those from Brazil, Africa and Europe. On the other hand, BH46 strain (type III LPG) is only found in $5 \%$ of the strains ${ }^{13}$.

COX-2 is involved in L. infantum LPG-induced PGE 2 production. Since intact $L$. infantum LPG from the BA262 strain was capable of inducing $\mathrm{PGE}_{2}$ production in IFN- $\gamma$ BMDM (Fig. 1A-D), COX-2 inhibition was performed by pretreatment of cells with NS-398, a selective COX-2 inhibitor, which blocked LPG-induced PGE 2 production (Fig. 2A). In contrast, LDs formation (Fig. 2B) and NO production (Fig. 2C) were shown to be unaffected by NS-398 treatment, suggesting that COX-2 activity is not required for LD formation or NO production.

Intact $L$. infantum BA262-derived LPG induces MAPK phosphorylation and a proinflammatory response. Leishmania sp. LPG has been shown to rapidly induce MAP kinase activation ${ }^{18,21}$, which was confirmed herein by ERK and JNK activation via Western blotting of BMDMs stimulated with intact L. infantum LPG (Fig. 3). We then verified if the inhibition of ERK1/2 (by PD98059) and PKC (by BIS I) would influence the release of LPG-induced proinflammatory mediators. LPG stimulation alone was shown to induce PGE $_{2}, \mathrm{MCP}^{-1}$, IL-6, TNF- $\alpha$ and IL-12p70 production (Fig. 4), but did not affect levels of IL-10. When ERK phosphorylation was inhibited, reduced LPG induced-production of PGE 2 , MCP-1, TNF- $\alpha$ and IL-12p70 was observed. Furthermore, 

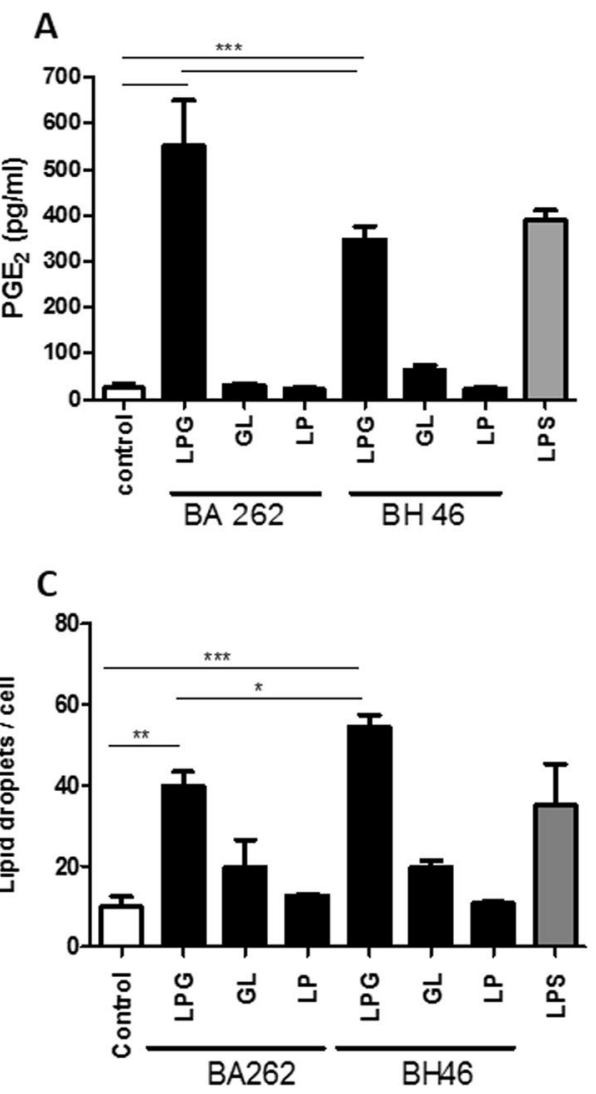

B

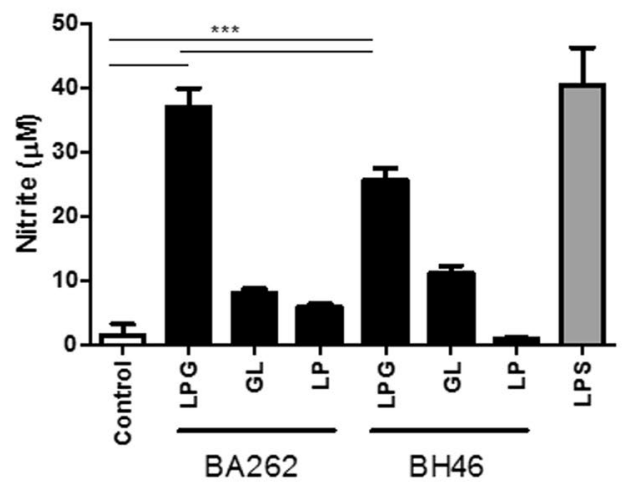

D

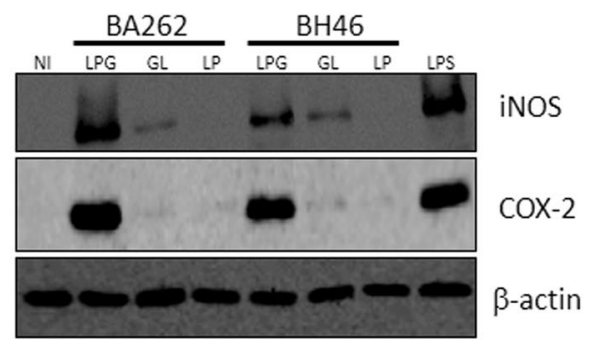

Figure 1. Intact LPG extracts induce a prostaglandin E2-associated inflammatory response. Bone Marrow Derived Macrophages (BMDM) primed with IFN- $\gamma(100 \mathrm{ng} / \mathrm{mL})$ were stimulated for $24 \mathrm{~h}$ with $10 \mu \mathrm{g} / \mathrm{mL}$ of intact LPG extract or LPG-derived fragments: lipids (LP) or glycans (GL) from the BA262 or BH46 strains. LPS $(500 \mathrm{ng} / \mathrm{ml})$ was used as a positive control. $\mathrm{PGE}_{2}(\mathbf{A})$ and $\mathrm{NO}(\mathbf{B})$ were measured in culture supernatants. $(\mathbf{C})$ Lipid droplets were enumerated on slides stained with osmium tetroxide. Bars represent means \pm SD of three representative experiments performed in triplicate. ANOVA test followed by Student Newman-Keuls post-test was used to multiple comparison among experimental groups (*p $<0.05$, **p $<0.01$ and $* * * p<0.001)$. (D) iNOS and COX-2 induction were evaluated by Western Blot $24 \mathrm{~h}$ after LPG stimulation.

PKC inhibition reduced TNF- $\alpha$ and IL-12p70 production. Interestingly, the inhibition of both signaling pathways resulted in decreased IL-6 production in the presence of LPG, yet IL-10 was unaffected (Fig. 4).

PPAR- $\gamma$ regulates TLR1/2 activation by LPG stimulation. Since LPG has been shown to activate TLR$1 / 2^{21,28,37}$, we employed a luciferase reporter assay using HEK293 cells transfected with different combinations of TLR-1 and -2, including PPAR- $\gamma$ to confirm the downstream response. TLR1 and TLR2 were found to be activated in the presence of L. infantum LPG and PamCys3 (a TLR-1/2 agonist, positive control) (Fig. 5A). TLR1/2 activation was able to induce PPAR- $\gamma$ expression (Fig. 5B). These findings indicate that TLR1/2 recognize L. infantum LPG with further downstream regulation by PPAR- $\gamma$. Next, to investigate the potential role of PPAR- $\gamma$ in the regulation of an LPG-induced inflammatory response in activated macrophages, cells were incubated in the presence or absence of rosiglitazone, a PPAR- $\gamma$ agonist, prior to the addition of purified LPG (Fig. 6). PPAR- $\gamma$ activation decreased the levels of PGE 2 , MCP-1, IL-6, TNF- $\alpha$ and IL12p70, whereas IL-10 production remained unaffected (Fig. 6). These data reinforce the role of PPAR- $\gamma$ activation in the downregulation of an LPG-induced inflammatory response.

\section{Discussion}

Glycoconjugates are important PAMPs in trypanosomatids that play pivotal roles during parasite-host interaction. In this context, LPG is a key virulence factor expressed at the surface of Leishmania promastigotes. Many functions have been attributed to this molecule in both vertebrate and invertebrate hosts ${ }^{15,16,38,39}$. While the roles of LPG in the innate immune response have been well established, the literature contains scarce data regarding the involvement of LPGs in lipid mediator production. Previous studies by our group have shown that $L$. amazonensis-derived LPG induced $\mathrm{LTB}_{4}$ production by human neutrophils ${ }^{23}$. Here we investigated whether the LPGs and/or LPG-derived moieties from different L. infantum strains were capable of triggering $\mathrm{PGE}_{2}$ and proinflammatory cytokine production.

While comprehensive knowledge surrounding the critical aspects of LPG structure and functioning remains limited, one distinguishing feature of L. infantum-derived LPG is that it exhibits intraspecies polymorphisms (type I, II and III). Since the number of $\beta$-glucose residues branching off the repeat units is critical to NO 
A

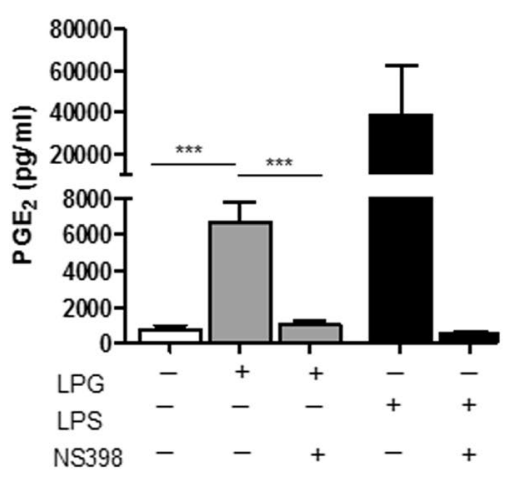

B

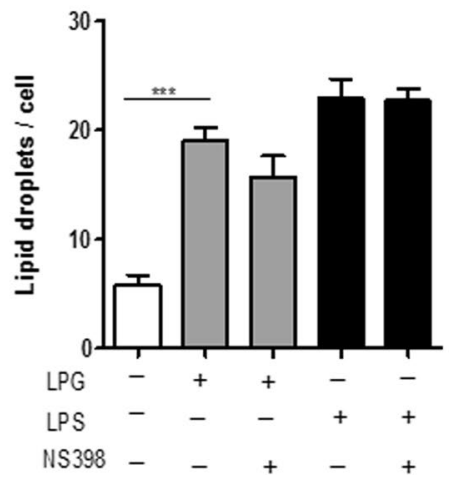

C

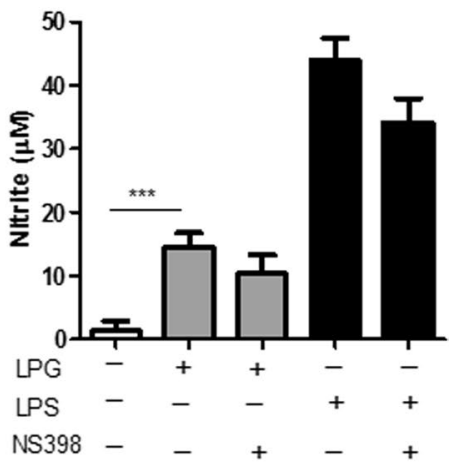

Figure 2. NS398 inhibits L. infantum LPG-induced PGE2 production, but did not alter lipids droplet formation or nitric oxide production. IFN- $\gamma$-primed BMDM were preincubated for $1 \mathrm{~h}$ with NS-398 $(1 \mu \mathrm{M})$, a specific COX-2 inhibitor, then stimulated for $24 \mathrm{~h}$ with $10 \mu \mathrm{g} / \mathrm{mL}$ purified intact LPG extract from the BA262

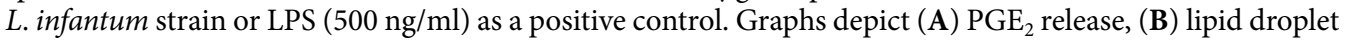
quantification and $(\mathbf{C})$ nitric oxide production. Bars represent means \pm SD of two representative experiments performed in quadruplicate. ANOVA test followed by Student Newman-Keuls post-test was used to multiple comparison among experimental groups $(* * * \mathrm{p}<0.001)$.

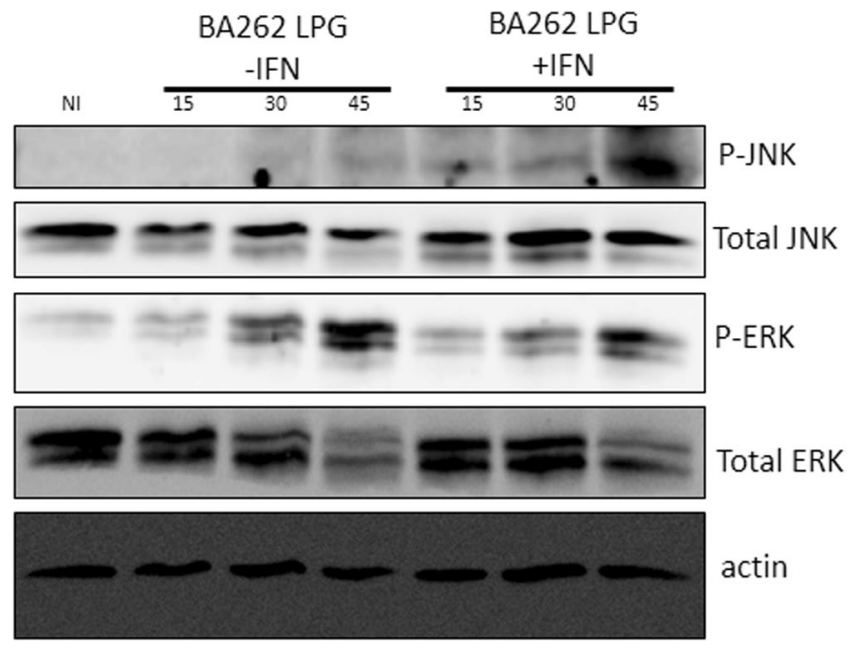

Figure 3. L. infantum LPG induces rapid ERK1/2 and JNK phosphorylation. BMDM were primed or not with IFN- $\gamma(100 \mathrm{ng} / \mathrm{mL})$ and stimulated with $10 \mu \mathrm{g} / \mathrm{mL}$ of purified intact LPG extract from the BA262 strain for 15, 30 and $45 \mathrm{~min}$. Bands indicate ERK1/2 and JNK phosphorylation in cell lysates evaluated by Western Blot.

production $^{13}$, we purified LPGs from L. infantum strains BA262 (type I) and BH46 (type III), as well as their lipid and glycan motifs. Consistent, only intact LPG from both $L$. infantum strains was shown to trigger robust nitrite production and iNOS expression by murine macrophages.

Glycoconjugates are GPI-anchored structures that may be released in the extracellular milieu either due to the action of different phospholipases, or as surface components of extracellular vesicles ${ }^{40-42}$. These include LPGs, GIPLs, proteophosphoglycans (PPGs), phosphoglycans (PGs), secreted acid phosphatases (sAP) and glycoprotein 63 (GP63) $^{43}$. In the case of L. major LPG and Trypanosoma cruzi glycosylphosphatidylinositol (GPI)-mucins, disruption of the lipid anchor was shown to abrogate proinflammatory activity and NO production ${ }^{19,44}$. In addition, treatment of L. braziliensis GIPLs with phosphatidylinositol-specific phospholipase C (PI-PLC) was shown to restore NO production by murine macrophages ${ }^{16}$. In the other hand, here the treatment of LPG from both $L$. infantum strains with phosphatidylinositol-specific phospholipase C (PI-PLC) decreased levels of NO, PGE $_{2}$, lipid body formation and COX-2 expression by macrophages. By contrast, the diminished action evidenced by the purified lipid and glycan moieties as compared to that of intact LPG, suggests that these fragments may act synergistically. Another possibility could be that the lipid anchor is required for the insertion of the highly-charged phosphoglycan moiety into the membrane.

Although both intact LPG from BA262 and BH46 induced a proinflammatory response, PGE and NO induction were higher in the supernatant of macrophages stimulated with BA262 LPG, while droplet formation increased in cells stimulated with BH46 LPG. This finding may be explained by the fact that lipid droplets serve as reservoirs that provide substrate for the enzymatic activity of COX-2 (which produces $\mathrm{PGE}_{2}$ ), as 
A

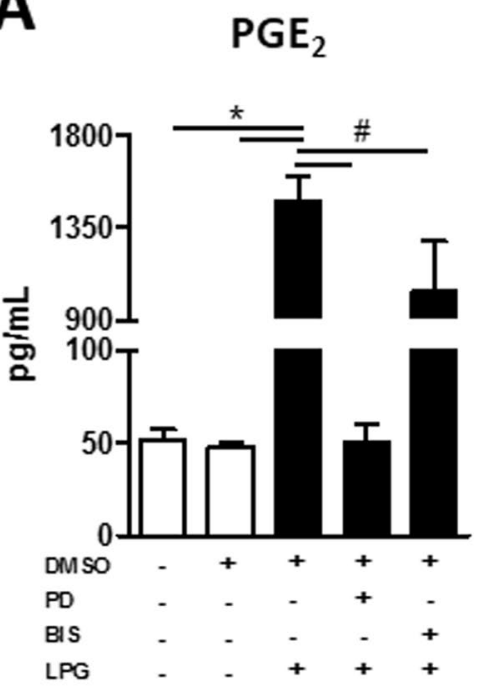

D

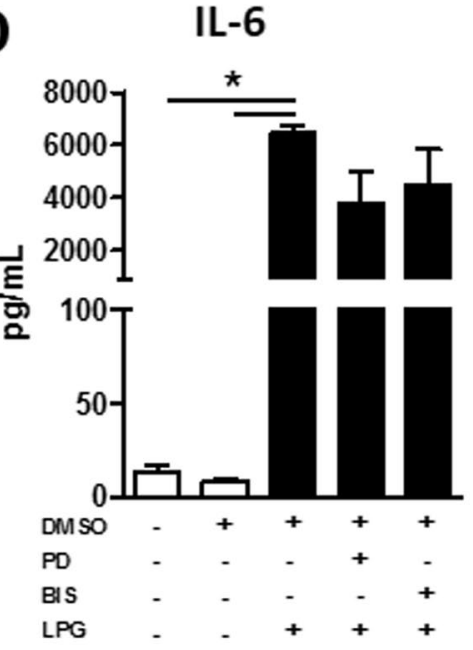

B
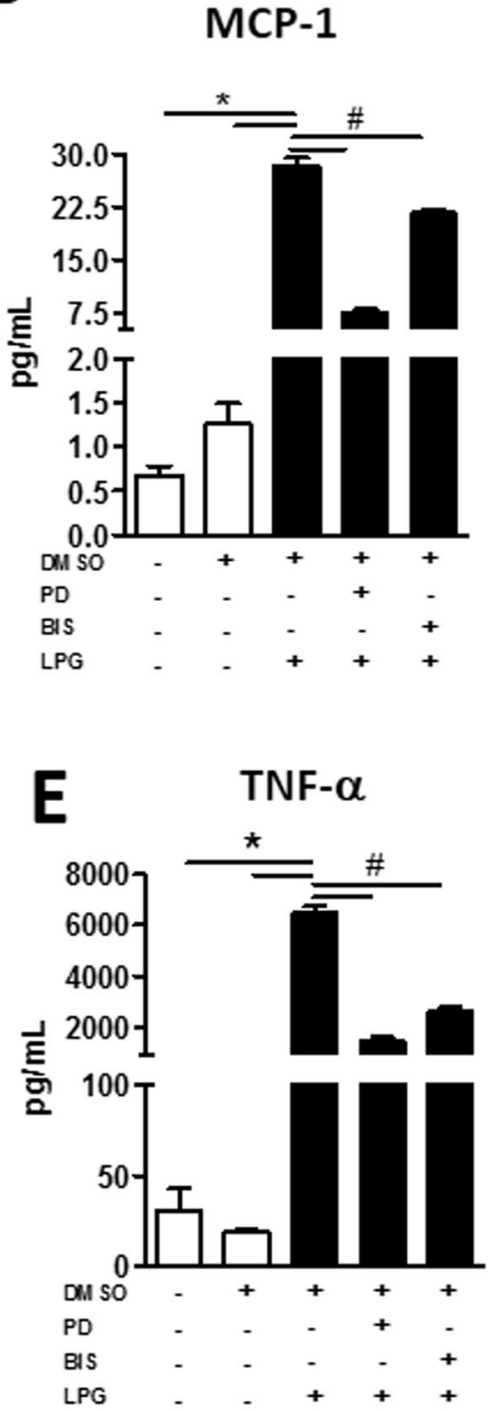

IL-10

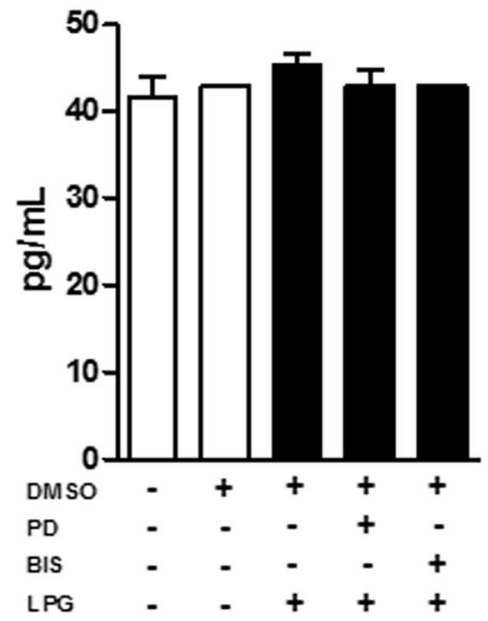

Figure 4. ERK-1/2 and PKC signaling induced by BA262 L. infantum LPG drives PGE2 and proinflammatory mediator production. Murine macrophages were pretreated for $1 \mathrm{~h}$ with $50 \mu \mathrm{M}$ PD98059 (ERK-1/2 inhibitor) or $20 \mu \mathrm{M}$ BIS I (PKC inhibitor) and stimulated for $24 \mathrm{~h}$ with $10 \mu \mathrm{g} / \mathrm{mL}$ purified intact LPG extract from the BA262 strain. (A) PGE 2 , (B) MCP-1, (C) IL-10, (D) IL-6, (E) TNF- $\alpha$, (F) IL-12p70 levels were measured in culture supernatant. Bars represent means $\pm \mathrm{SD}$ of two representative experiments in quintuplicate. ANOVA test followed by Student Newman-Keuls post-test was used to multiple comparison among experimental groups $\left(* \mathrm{p}<0.05\right.$ compared to the control group; ${ }^{*} \mathrm{p}<0.05$ compared to other groups).

well as 5-lipoxigenase (5-LO, which produces leukotrienes and lipoxins) ${ }^{45}$. In addition, the utilization of lipid droplet-derived arachidonic acid by both COX-2 or 5-LO is not well understood. Accordingly, it is possible that discrepancies between lipid droplet quantification and $\mathrm{PGE}_{2}$ measurements results from the activation of enzymatic pathways other than COX-2. Interestingly, treatment with NS-398, an irreversible COX-2 inhibitor, was shown to reduced $\mathrm{PGE}_{2}$ levels, yet LD formation and NO production remained unaffected, which suggests that the enhanced LD formation induced by BH46 LPG is unrelated to NO or PGE2 production. This reinforces the notion that LPG integrity is functionally required for the immunomodulation of host cells.

A diverse range of PAMPs and DAMPs can activate ERK-1/2 and JNK signaling pathways ${ }^{46-48}$. Early studies using synthetic Leishmania PGs demonstrated the effect of this glycoconjugate in subverting IL-12 production via the ERK pathway ${ }^{49}$. Later, LPG-induced MAPKs were investigated in other New World Leishmania species ${ }^{17,21}$. The ability of LPGs to induce MAPKs is variable, and is perhaps species-specific. For example, LPGs derived from L. mexicana, L. infantum (BH46 strain), L. enriettii, L. braziliensis and L. amazonensis were shown to differentially activate ERK-1/2, p38 and JNK pathways ${ }^{17,18,21,37}$. On the other hand, GIPLs from L. infantum and L. braziliensis are potent inhibitory molecules that do not activate ERK, p38 and JNK ${ }^{16}$. Here, LPG from L. infantum BA262 was shown to induce ERK-1/2 and JNK phosphorylation in macrophages, which confirms that the mechanisms underlying MAPKs pathways participate in signaling following stimulation with glycoconjugates. Indeed, the 
A

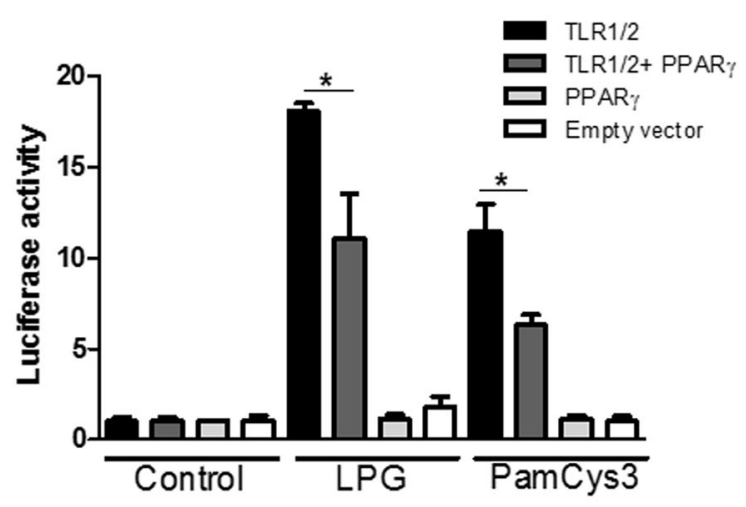

B

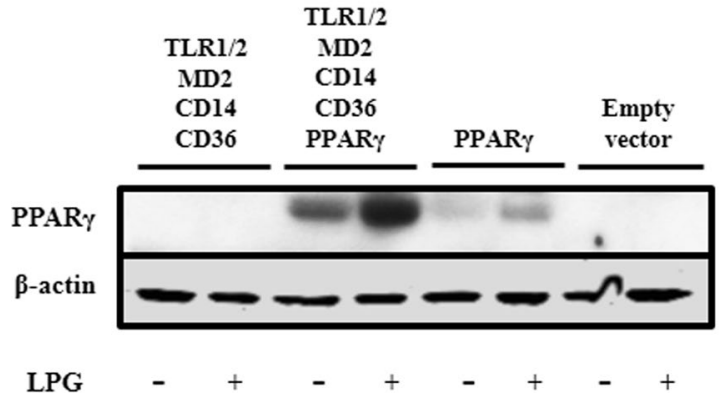

Figure 5. TLR-1/2-induced PPAR- $\gamma$ signaling is trigged by BA262 L. infantum LPG (A) HEK 293 T cells were transfected with four different constructions: (i) TLR-1/2, MD2, CD14 and CD36; (ii) TLR-1/2, MD2, CD14, CD36 and PPAR- $\gamma$; (iii) PPAR- $\gamma$ transfection control; (iv) empty vector. After $24 \mathrm{~h}$ of transfection, cells were stimulated with $10 \mu \mathrm{g} / \mathrm{mL}$ of purified intact LPG extract from the BA262 strain, $1 \mathrm{nM}$ of Pam3CSK4 or medium alone (control). After $4 \mathrm{~h}$, transfected HEK $293 \mathrm{~T}$ cells were lysed and luciferase activity was assessed by NF- $\kappa \mathrm{B}$ activation using a Dual-Luciferase Reporter Assay System. Bars represent means \pm SD of two representative experiments performed in triplicate. ANOVA test followed by Tukeys' post-test was used to multiple comparison among experimental groups $(* \mathrm{p}<0.5)$. (B) PPAR- $\gamma$ expression was evaluated by western blotting after LPG stimulation in the four groups of transfected HEK 293 T cells using $\beta$-actin as a housekeeping gene.

pharmacologic inhibition of PKC and ERK-1/2 signaling was found to affect the production of some cytokines and $\mathrm{PGE}_{2}$ under stimulation with intact LPG.

Similarly to MAPKs, the secretion of proinflammatory cytokines and chemokines varies greatly among different Leishmania species, not only in the Old World, but also in species found in the New World. Inflammatory signaling and cytokine production are essential to parasite control. During Leishmania infection, a proinflammatory response that results in tissue damage could be triggered by LPG or other molecules secreted by the parasite ${ }^{50-53}$. Thus, the mechanisms underlying immune response activation by Leishmania PAMPs is crucial in determining the inflammatory balance that defines whether an infection will be controlled or exacerbated ${ }^{54}$. Interestingly, following LPG stimulation, dermotropic species exhibit a more exacerbated proinflammatory profile, whereas viscerotropic species present immunosuppression. This is the case with L. infantum LPG which was not able to translocate NF-kB (strain BH46), and induced lower levels of NO and cytokines (TNF- $\alpha$, IL-1 $\beta$ and IL-6) compared to L. braziliensis, ${ }^{21}$. Here, LPG from strain BA262 successfully induced cytokine and chemokine production by murine macrophages.

It is interesting to note that a COX-2 antagonist was not capable of reducing LD formation and NO production, which indicates that other pathways may be involved in LPG-induced $\mathrm{PGE}_{2}$ production. PPAR- $\gamma$ signaling has been shown to coordinate the inflammatory immune response, as well as COX-2 activation and the induction of lipid mediators in a variety disease model $s^{36,55-61}$. The activation of PPAR- $\gamma$ by intracellular mycobacterial infection induces lipid droplet formation and $\mathrm{PGE}_{2}$ production, as well as favoring mycobacterial survival through the downmodulation of NF- $\kappa$ B signaling ${ }^{35,62}$. Similarly, L. donovani infection induces PPAR- $\gamma$ activation and promotes parasite survival, whereas the inhibition of PPAR- $\gamma$ facilitates Leishmania clearance ${ }^{63}$. By contrast, PPAR- $\gamma$ activation in L. mexicana-infected macrophages was shown to control parasite burden by oxidative stress, while selectively regulating prostaglandin production, yet with no reduction in $\mathrm{PGE}_{2}$ production following infection ${ }^{36}$. On the other hand, L. infantum infection or stimulation by LPG induced the production of heme oxygenase 1 (HO-1) in murine macrophages in a manner not dependent on PPAR- $\gamma^{64}$. The present results using a HEK luciferase reporter systems evidence that PPAR- $\gamma$ is induced by L. infantum intact LPG. In addition, an LPG-induced inflammatory response was successfully reverted by PPAR- $\gamma$ activation. Rosiglitazone, a PPAR- $\gamma$ agonist was shown to reduce the production of $\mathrm{PGE}_{2}$ and $\mathrm{MCP}-1$, in addition to a range of pro-inflammatory cytokines, suggesting the involvement of a mechanism other than NF- $\kappa B$ signaling. Although the significance of PPAR- $\gamma$ activation by L. infantum LPG is not fully understood, this induction could represent a strategy for the control of an LPG-mediated inflammatory response that is capable of inducing tissue damage during initial infection stages. 

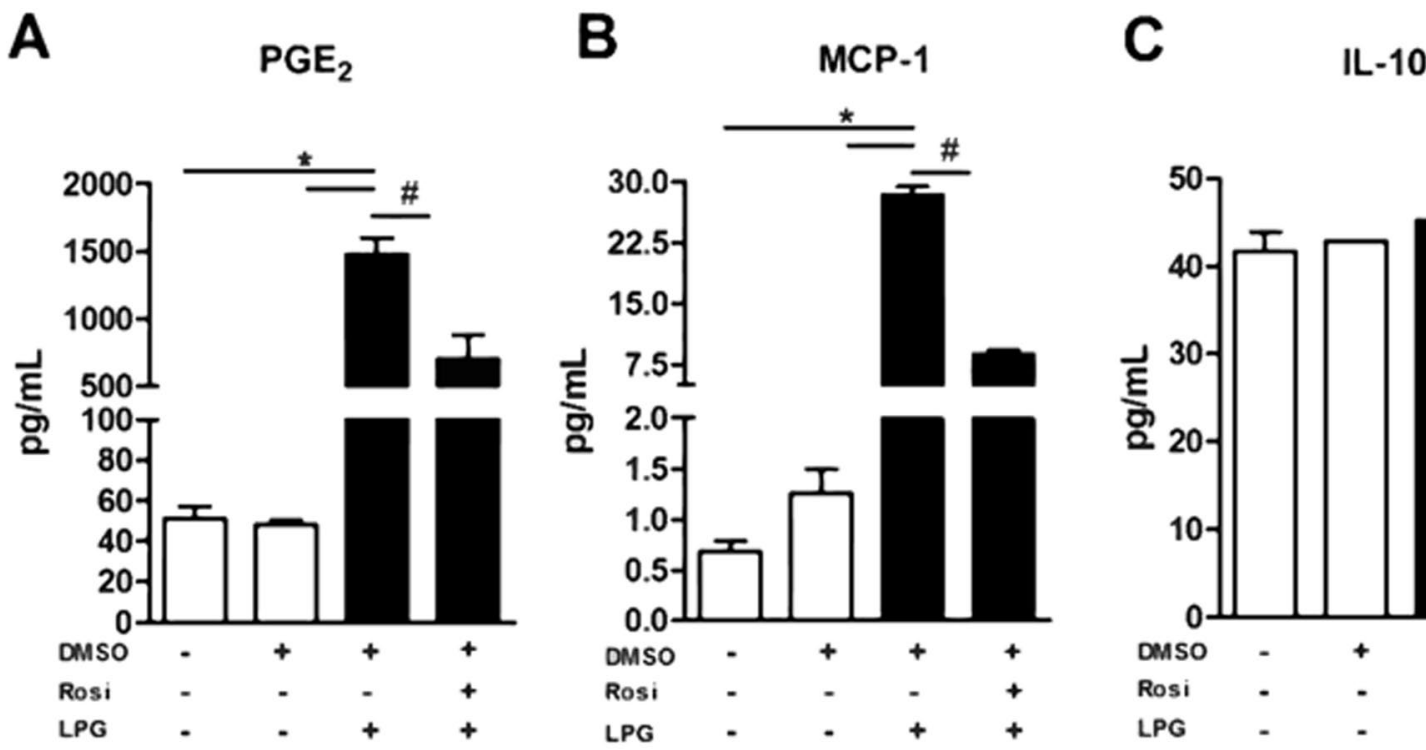

IL-10

D

E

\section{IL-6}

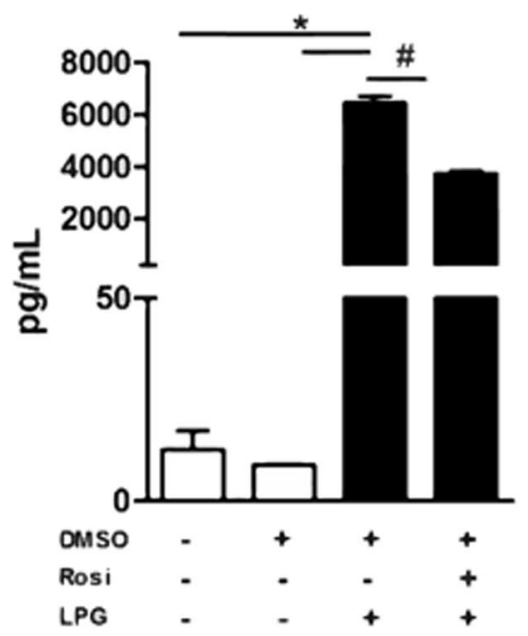

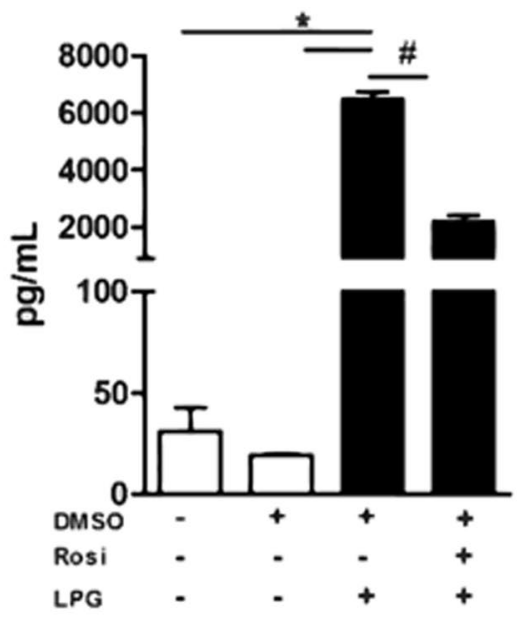

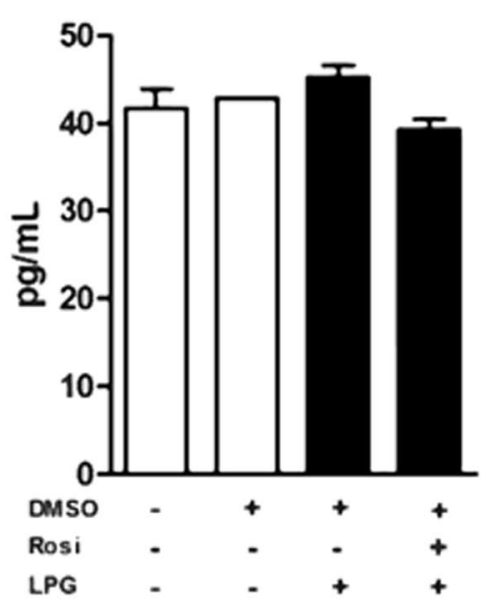

$\mathbf{F}$

IL-12

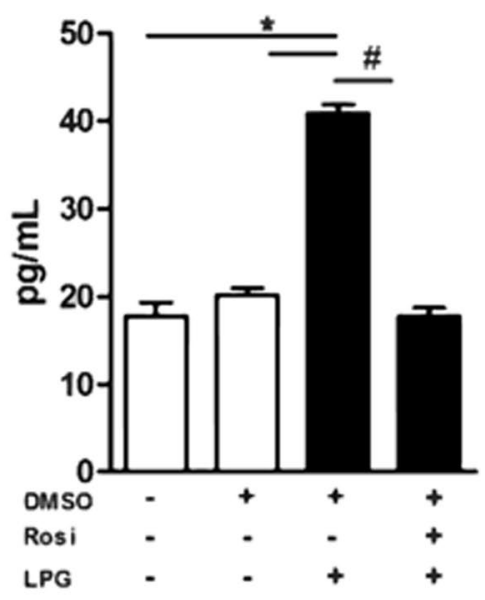

Figure 6. PPAR- $\gamma$ signaling induced by BA262 L. infantum LPG drives PGE2 and pro-inflammatory mediator production. Murine macrophages were treated for $24 \mathrm{~h}$ with $10 \mu \mathrm{M}$ rosiglitazone, a PPAR- $\gamma$ agonist $(10 \mu \mathrm{M})$, then stimulated for $24 \mathrm{~h}$ with $10 \mu \mathrm{g} / \mathrm{mL}$ of purified intact LPG extract from the BA262 strain. Levels of (A) PGE2, (B) MCP-1, (C) IL-10, (D) IL-6, (E) TNF- $\alpha$, (F) IL-12p70 were measured in culture supernatant. Bars represent means $\pm \mathrm{SD}$ of two representative experiments in quintuplicate. ANOVA test followed by Student Newman-Keuls post-test was used to multiple comparison among experimental groups $(* \mathrm{p}<0.05$ compared to the control group; ${ }^{\mathrm{p}}<0.05$ compared to other groups).

Based on the present results, we have developed an illustrative model (Fig. 7) to explain the involvement of TLR1/2 in the recognition of L. infantum LPG. A previous investigation revealed that while TLR2 participates in the induction of $\mathrm{PGE}_{2}$ by Leishmania, the identity of the ligand(s) involved in this activation remains undetermined ${ }^{28}$. Using a HEK293 reporter cell system, we have demonstrated that intact LPG is capable of activating TLR-1/2 consequently inducing the expression of PPAR- $\gamma$. Finally, the TLR-1/2 activation by LPG engages kinase-signaling proteins, such as PKC, ERK1-2 and JNK, that contribute to a $\mathrm{PGE}_{2}$-associated inflammatory response. In parallel, as PPAR- $\gamma$ partially regulates inflammatory activation, this reinforces the notion that PPAR- $\gamma$ is a critical regulator of LPG-induced inflammatory signaling. Further study is needed to more comprehensively address the role played by LPG in the outcome of L. infantum infection.

\section{Methods}

Antibodies and Reagents. RPMI 1640 medium and L-glutamine, penicillin, and streptomycin were obtained from Invitrogen (Carlsbad, CA, USA). Nutridoma-SP was purchased from Roche (Indianapolis, In, USA). NS-398, Rosiglitazone and the $\mathrm{PGE}_{2}$ enzyme-linked immunoassay (EIA) kit were acquired from Cayman 


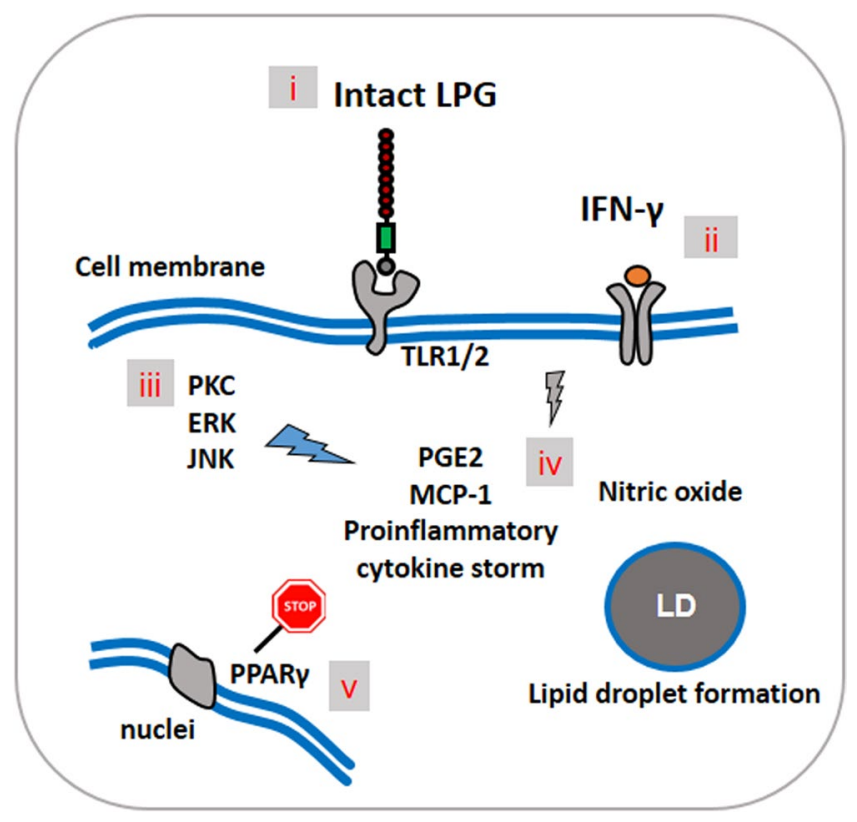

Figure 7. Illustration of $L$. infantum LPG-induced $\mathrm{PGE}_{2}$-associated inflammatory response. Intact LPG is recognized by TLR-1/2 (i) on the membranes of IFN- $\gamma$ - primed macrophages (ii), triggering the MAPs kinase signaling pathway, leading to the phosphorylation of PKC, ERK and JNK (iii). This process induce thes $\mathrm{PGE}_{2}$ production and lipid droplet formation, as well as $\mathrm{NO}$ and $\mathrm{MCP}-1$ production in addition to a proinflammatory cytokine storm (iv). PPAR $\gamma$ regulates the LPG-induced $\mathrm{PGE}_{2}$-associated inflammatory response in a feedback mechanism (v). Illustration by Théo Araújo-Santos.

Chemical (Ann Arbor, MI, USA). Osmium tetroxide $\left(\mathrm{OsO}_{4}\right)$ was obtained from Electron Microscopy Science (Fort Washington, PA, USA). AquaPolymount was purchased from Polysciences (Warrington, PA, USA). PD 98059, 29-Amino-39-methoxyflavone and Bisindolylmaleimide-I, 2-[1-(3-Dimethylaminopropyl)-1H-indol-3yl]-3-(1H-indol-3-yl)-maleimide (BIS) were obtained from Merck-Calbiochem (Darmstadt, Hessen, Germany). S-[2,3-bis(palmitoyloxy)-(2-RS)-propyl]-N-palmitoyl-(R)-Cys-Ser-Lys4-OH (PamCys3), a TLR2/TLR1 agonist, was acquired from Invitrogen (San Diego, CA, USA) and a Bead Array mouse inflammation kit was purchased from BD Biosciences (San Jose, CA, USA). Primary $\beta$-actin, pERK, ERK, P-JNK and JNK antibodies were obtained from Cell Signaling (Danvers, MA, USA), while Cox-2 and iNOS antibodies were purchased from Calbiochem (San Diego, CA, USA). The Dual-Luciferase Reporter Assay System was acquired from Promega (Madison, WI, USA).

Ethics Statement. All experiments were performed in strict accordance with the recommendations of the Brazilian Council for the Control of Animal Experimentation (CONCEA). The Oswaldo Cruz Foundation Institutional Review Board for Animal Experimentation (CEUA-IGM-FIOCRUZ) approved all present experimental protocols (Protocol number: 021/2015).

Animals. Inbred male C57BL/6 mice aged 6-8 weeks were obtained from the animal care facility at the Gonçalo Moniz Institute, Oswaldo Cruz Foundation (IGM-FIOCRUZ, Bahia-Brazil). The animals were kept at a temperature of $24^{\circ} \mathrm{C}$ with free access to food and water under regular 12-hour cycles of light and darkness.

Leishmania infantum culture and LPG Extraction. L. infantum (strains MCAN/BR/89/BA262 and MHOM/BR/1970/BH46) promastigotes were cultured in M199 medium supplemented with 10\% inactive FBS, 2 $\mathrm{mM}$ L-glutamine, $100 \mathrm{U} / \mathrm{ml}$ penicillin, $50 \mu \mathrm{g} / \mathrm{ml}$ streptomycin, $12.5 \mathrm{mM}$ glutamine, $0.1 \mathrm{M}$ adenine, $0.0005 \%$ hemin and $40 \mathrm{mM}$ Hepes, adjusted to a $\mathrm{pH} 7.4$, at $26^{\circ} \mathrm{C}$ until late log-phase. LPG was extracted and purified as previously described $^{65}$. LPG purity was checked using Chinese hamster ovary (CHO) cells transfected with TLR2 or TLR4, as reported elsewhere ${ }^{17}$. Purified LPG extract was treated with Phosphatidylinositol-specific phospholipase C (PI-PLC) to release the lipid and glycan moieties. Glycans and lipids were separated as previously described ${ }^{66}$.

Macrophage culturing and IFN- $\gamma$ activation. Bone Marrow Derived Macrophages (BMDM) were obtained from C57BL/6 mice. Cells were collected from femurs and differentiated in RPMI 1640, 20\% FBS, 30\% L-929 cell-conditioned media (LCCM), $2 \mathrm{mM} \mathrm{L}$-glutamine and $100 \mathrm{U} / \mathrm{mL}$ penicillin/Streptomycin at $36^{\circ} \mathrm{C}$ under $5 \% \mathrm{CO}_{2}$. BMDMs were collected after seven days and seeded on tissue culture plates in RPMI 1640 media, 10\% FBS, 5\% LCCM and $2 \mathrm{M} \mathrm{L}$-glutamine. For cytokine quantification, peritoneal macrophages were obtained four days after intraperitoneal injection of $1 \mathrm{~mL} 3 \%$ thioglycolate solution in C57BL/6 mice. Peritoneal cells were harvested using $10 \mathrm{~mL}$ RPMI 1640 and then centrifuged at $400 \mathrm{xg}$ for 10 minutes. Macrophages $\left(3 \times 10^{5} /\right.$ well $)$ were cultured in $1 \mathrm{~mL}$ of RPMI 1640 medium supplemented with $2 \mathrm{mM} \mathrm{L}$-glutamine, $100 \mathrm{U} / \mathrm{mL}$ penicillin and $100 \mu \mathrm{g} /$ 
$\mathrm{mL}$ streptomycin. For activation, all macrophage cultures were stimulated with $100 \mathrm{U} / \mathrm{mL}$ recombinant IFN- $\gamma$ for 24 hours. IFN- $\gamma$-primed macrophages were used in experimentation as unprimed cultures stimulated with LPG fail to induce $\mathrm{NO}^{13}$.

$\mathrm{PGE}_{2}$, $\mathrm{NO}$ and lipid droplet quantification. $\mathrm{PGE}_{2}$ and $\mathrm{NO}$ was evaluated in the culture supernatants of IFN- $\gamma$-primed BMDM treated for $24 \mathrm{~h}$ with $10 \mu \mathrm{g} / \mathrm{mL}$ intact LPG extract or LPG-derived fragments (lipids or glycans) from the BA262 or BH46 strains. LPS $(500 \mathrm{ng} / \mathrm{ml})$ was used as a positive control. Levels of PGE . were measured by EIA in the supernatant in accordance with manufacturer protocols. Nitrite concentrations were determined by Griess reaction. Lipid droplet quantification was performed in adhered cells fixed in $3.7 \%$ formaldehyde, then stained with osmium tetroxide as described previously ${ }^{67}$. Lipid droplets were counted by light microscopy using a 100x objective lens in 50 consecutively scanned macrophages. For the COX-2 enzyme inhibition assays, IFN- $\gamma$-primed BMDM were preincubated for $1 \mathrm{~h}$ with NS-398 $(1 \mu \mathrm{M})$, a specific COX-2 inhibitor. Next, cells were stimulated with $10 \mu \mathrm{g} / \mathrm{mL}$ purified intact LPG extract only from the BA262 L. infantum strain, or LPS (500 ng/ml) as a positive control. After $24 \mathrm{~h}, \mathrm{PGE}_{2}, \mathrm{NO}$ and lipid droplet quantification was performed as described above.

Signaling pathway inhibition. For the inhibition assays, IFN- $\gamma$-primed peritoneal macrophages were preincubated for $1 \mathrm{~h}$ with: i) $20 \mu \mathrm{M}$ BIS, an inhibitor of PKC; ii) $50 \mu \mathrm{M}$ PD98059, an inhibitor of ERK-1/2; iii) $10 \mu \mathrm{M}$ Roziglitazone, an agonist of PPAR- $\gamma$. Next, cells were stimulated for $24 \mathrm{~h}$ with purified intact $10 \mu \mathrm{g} / \mathrm{mL} \mathrm{LPG}$ extract from only the BA262 L. infantum strain. $\mathrm{PGE}_{2}$ levels were measured in the supernatant as described above. Cytokines (TNF- $\alpha$, IL-6 MCP-1, IL-12p70 and IL-10) were quantified using a Cytometric Bead Array (CBA) inflammatory kit mouse following manufacturer's instructions.

Western blot analysis. To evaluate the inflammatory response triggered by LPG stimulation, COX-2 and iNOS expression were determined by western blotting. IFN- $\gamma$-primed BMDM were stimulated with purified intact LPG extract or LPG-derived fragments from BA262 and BH46 L. infantum strains for 24h. BMDMs were then lysed in RIPA buffer supplemented with a cocktail of protease and phosphatase inhibitors. Protein extracts were resolved by SDS-PAGE and transferred to a nitrocellulose membrane, then probed with Cox- 2 and iNOS. Levels of phosphorylated MAPKs signaling pathways triggered by LPG stimulation were also examined by western blotting. BMDMs, primed or not with IFN- $\gamma$, were stimulated with $10 \mu \mathrm{g} / \mathrm{mL}$ purified intact LPG extract only from the BA262 strain for 15, 30 or 45 min., then probed with $\beta$-actin, pERK, ERK, P-JNK and JNK. Bands were detected using an Image Quant LAS 4010 (GE healthcare).

HEK 293T cell transfection assay. For the transfection assays, HEK $293 \mathrm{~T}$ cells were plated in 12 -well plates $\left(5 \times 10^{5}\right.$ cells/well) after $24 \mathrm{~h}$ using Lipofectamine 2000 and Opti-MEM (Invitrogen) in accordance with manufacturer instructions. Four different groups were transfected: (1) TLR2, TLR1, MD2, CD14 and CD36; (2) TLR2, TLR1, MD2, CD14, CD36 and PPAR $\gamma$; (3) PPAR $\gamma$ transfection control; (4) empty vector. For transfection, the following targets were added to the wells: $0.2 \mu \mathrm{g}$ mouse TLR2, $0.8 \mu \mathrm{g}$ mouse TLR1, $0.22 \mu \mathrm{g}$ mouse MD-2,

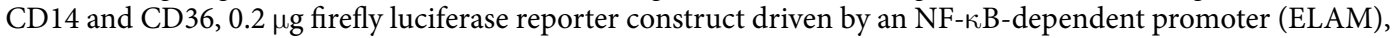
$6.6 \mathrm{ng}$ of the Renilla luciferase reporter construct ( $\beta$-actin-Renilla luciferase, used as a control for transfection efficiency), and varying amounts of PDisplay (an empty vector) to reach a final volume of $2 \mu \mathrm{g}$ of DNA in each well. After $24 \mathrm{~h}$ of transfection, cells were then stimulated for $4 \mathrm{~h}$ with $10 \mu \mathrm{g} / \mathrm{mL}$ of purified intact LPG extract, $1 \mathrm{nM}$ of PamCys 3 or medium alone (control samples). Finally, transfected HEK 293 T cells were lysed and luciferase activity was assessed by NF- $\kappa$ B activation using the Dual-Luciferase Reporter Assay System in accordance with manufacturer instructions. Relative luminescence units (RLU) were quantitated using a SpectraMaxL luminometer. The values obtained for stimulated cells were relativized using values from non-stimulated cells. Luciferase activity is expressed as the ratio of NF- $\kappa \mathrm{B}$-dependent firefly luciferase activity to constitutively expressed Renilla luciferase activity. PPAR- $\gamma$ expression was evaluated by western blotting after LPG stimulation in the four groups of transfected HEK 293 T cells using $\beta$-actin as a housekeeping gene.

Statistical analysis. All assays were performed using at least three replicates per group and each experiment was performed in triplicate. Data, presented as means and SE (standard error) from representative experiments, were analyzed using GraphPad Prism 5.0 software (GraphPad Software, San Diego, CA, USA). Comparisons between groups were analyzed using the ANOVA with Student Newman-Keuls multiple comparison was used as post-hoc test. HEK 293 reporter assay where analyzed by ANOVA followed by Tukey multiple comparison post-hoc test. Differences were considered statistically significant when $\mathrm{p} \leq 0.05$.

\section{References}

1. Herwaldt, B. L. Leishmaniasis. Lancet 354, 1191-1199 (1999)

2. de Assis, R. R., Ibraim, I. C., Nogueira, P. M., Soares, R. P. \& Turco, S. J. Glycoconjugates in New World species of Leishmania: polymorphisms in lipophosphoglycan and glycoinositolphospholipids and interaction with hosts. Biochim Biophys Acta 1820, 1354-1365 (2012).

3. Descoteaux, A., Turco, S. J., Sacks, D. L. \& Matlashewski, G. Leishmania donovani lipophosphoglycan selectively inhibits signal transduction in macrophages. J Immunol 146, 2747-2753 (1991).

4. Giorgione, J. R., Turco, S. J. \& Epand, R. M. Transbilayer inhibition of protein kinase C by the lipophosphoglycan from Leishmania donovani. Proc Natl Acad Sci USA 93, 11634-11639 (1996)

5. Desjardins, M. \& Descoteaux, A. Inhibition of phagolysosomal biogenesis by the Leishmania lipophosphoglycan. J Exp Med 185, 2061-2068 (1997).

6. Cunningham, A. C. Parasitic adaptive mechanisms in infection by leishmania. Exp Mol Pathol 72, 132-141 (2002).

7. Chawla, M. \& Vishwakarma, R. A. Alkylacylglycerolipid domain of GPI molecules of Leishmania is responsible for inhibition of PKC-mediated c-fos expression. J Lipid Res 44, 594-600 (2003). 
8. Delgado-Domínguez, J. et al. Leishmania mexicana lipophosphoglycan differentially regulates PKCalpha-induced oxidative burst in macrophages of BALB/c and C57BL/6 mice. Parasite Immunol 32, 440-449 (2010).

9. Kar, S., Ukil, A., Sharma, G. \& Das, P. K. MAPK-directed phosphatases preferentially regulate pro- and anti-inflammatory cytokines in experimental visceral leishmaniasis: involvement of distinct protein kinase C isoforms. J Leukoc Biol 88, 9-20 (2010).

10. Forestier, C. L., Gao, Q. \& Boons, G. J. Leishmania lipophosphoglycan: how to establish structure-activity relationships for this highly complex and multifunctional glycoconjugate? Front Cell Infect Microbiol 4, 193 (2014).

11. Sacks, D. L., Pimenta, P. F., McConville, M. J., Schneider, P. \& Turco, S. J. Stage-specific binding of Leishmania donovani to the sand fly vector midgut is regulated by conformational changes in the abundant surface lipophosphoglycan. J Exp Med 181, 685-697 (1995).

12. Soares, R. P. et al. Leishmania braziliensis: a novel mechanism in the lipophosphoglycan regulation during metacyclogenesis. Int $J$ Parasitol 35, 245-253 (2005).

13. Coelho-Finamore, J. M. et al. Leishmania infantum: Lipophosphoglycan intraspecific variation and interaction with vertebrate and invertebrate hosts. Int J Parasitol 41, 333-342 (2011).

14. Flandin, J. F., Chano, F. \& Descoteaux, A. RNA interference reveals a role for TLR2 and TLR3 in the recognition of Leishmania donovani promastigotes by interferon-gamma-primed macrophages. Eur J Immunol 36, 411-420 (2006).

15. Tuon, F. F. et al. Toll-like receptors and leishmaniasis. Infect Immun 76, 866-872 (2008).

16. Assis, R. R., Ibraim, I. C., Noronha, F. S., Turco, S. J. \& Soares, R. P. Glycoinositolphospholipids from Leishmania braziliensis and L. infantum: modulation of innate immune system and variations in carbohydrate structure. PLoS Negl Trop Dis 6, e1543 (2012).

17. Nogueira, P. M. et al. Lipophosphoglycans from Leishmania amazonensis Strains Display Immunomodulatory Properties via TLR4 and Do Not Affect Sand Fly Infection. PLoS Negl Trop Dis 10, e0004848 (2016).

18. Rojas-Bernabé, A. et al. Leishmania mexicana lipophosphoglycan activates ERK and p38 MAP kinase and induces production of proinflammatory cytokines in human macrophages through TLR2 and TLR4. Parasitology 141, 788-800 (2014).

19. de Veer, M. J. et al. MyD88 is essential for clearance of Leishmania major: possible role for lipophosphoglycan and Toll-like receptor 2 signaling. Eur J Immunol 33, 2822-2831 (2003).

20. Becker, I. et al. Leishmania lipophosphoglycan (LPG) activates NK cells through toll-like receptor-2. Mol Biochem Parasitol 130, 65-74 (2003).

21. Ibraim, I. C. et al. Two biochemically distinct lipophosphoglycans from Leishmania braziliensis and Leishmania infantum trigger different innate immune responses in murine macrophages. Parasit Vectors 6, 54 (2013).

22. Daugschies, a \& Joachim, a Eicosanoids in parasites and parasitic infections. Adv. Parasitol. 46, 181-240 (2000)

23. Tavares, N. M. et al. Understanding the mechanisms controlling Leishmania amazonensis infection in vitro: the role of LTB4 derived from human neutrophils. J Infect Dis 210, 656-666 (2014).

24. Afonso, L. et al. Interactions with apoptotic but not with necrotic neutrophils increase parasite burden in human macrophages infected with Leishmania amazonensis. J Leukoc Biol 84, 389-396 (2008).

25. Tavares, N. et al. Degranulating Neutrophils Promote Leukotriene B4 Production by Infected Macrophages To Kill Leishmania amazonensis Parasites. JImmunol 196, 1865-1873 (2016).

26. Araújo-Santos, T. et al. Prostaglandin E2/leukotriene B4 balance induced by Lutzomyia longipalpis saliva favors Leishmania infantum infection. Parasit Vectors 7, 601 (2014).

27. Brandonisio, O. et al. Nitric oxide production by Leishmania-infected macrophages and modulation by cytokines and prostaglandins. Parassitologia 43(Suppl 1), 1-6 (2001).

28. Bhattacharjee, A. et al. Leishmania donovani-Induced Prostaglandin E2 Generation Is Critically Dependent on Host Toll-Like Receptor 2-Cytosolic Phospholipase A2 Signaling. Infect Immun 84, 2963-2973 (2016).

29. França-Costa, J. et al. Arginase I, polyamine, and prostaglandin E2 pathways suppress the inflammatory response and contribute to diffuse cutaneous leishmaniasis. J Infect Dis 211, 426-435 (2015).

30. Malta-Santos, H. et al. Resolvin D1 drives establishment of Leishmania amazonensis infection. Sci Rep 7, 46363 (2017).

31. Araújo-Santos, T. et al. Anti-parasite therapy drives changes in human visceral leishmaniasis-associated inflammatory balance. Sci Rep 7, 4334 (2017).

32. Bozza, P. T., Bakker-Abreu, I., Navarro-Xavier, R. A. \& Bandeira-Melo, C. Lipid body function in eicosanoid synthesis: An update. Prostaglandins Leukot. Essent. Fat. Acids 85, 205-213 (2011).

33. Rodríguez, N. E. et al. Lipid bodies accumulation in Leishmania infantum-infected C57BL/6 macrophages. Parasite Immunol, doi:https://doi.org/10.1111/pim.12443 (2017).

34. Aleshin, S., Strokin, M., Sergeeva, M. \& Reiser, G. Peroxisome proliferator-activated receptor (PPAR) $\beta / \delta$, a possible nexus of PPAR $\alpha$ - and PPAR $\gamma$-dependent molecular pathways in neurodegenerative diseases: Review and novel hypotheses. Neurochemistry International 63, 322-330 (2013).

35. Almeida, P. E. et al. Differential TLR2 downstream signaling regulates lipid metabolism and cytokine production triggered by Mycobacterium bovis BCG infection. Biochim Biophys Acta 1841, 97-107 (2014).

36. Díaz-Gandarilla, J. A., Osorio-Trujillo, C., Hernández-Ramírez, V. I. \& Talamás-Rohana, P. PPAR activation induces M1 macrophage polarization via $\mathrm{CPLA}_{2}$-COX-2 inhibition, activating ROS production against Leishmania mexicana. Biomed Res Int 2013, 215283 (2013).

37. Paranaíba, L. F. et al. Leishmania enriettii: biochemical characterisation of lipophosphoglycans (LPGs) and glycoinositolphospholipids (GIPLs) and infectivity to Cavia porcellus. Parasit Vectors 8, 31 (2015).

38. Gazzinelli, R. T., Ropert, C. \& Campos, M. A. Role of the Toll/interleukin-1 receptor signaling pathway in host resistance and pathogenesis during infection with protozoan parasites. Immunol Rev 201, 9-25 (2004).

39. Moradin, N. \& Descoteaux, A. Leishmania promastigotes: building a safe niche within macrophages. Front Cell Infect Microbiol 2, 121 (2012).

40. Ferguson, M. A. The structure, biosynthesis and functions of glycosylphosphatidylinositol anchors, and the contributions of trypanosome research. J Cell Sci 112(Pt 1), 2799-2809 (1999).

41. Silverman, J. M. et al. An exosome-based secretion pathway is responsible for protein export from Leishmania and communication with macrophages. J Cell Sci 123, 842-852 (2010).

42. Campos, J. H. et al. Extracellular Vesicles: Role in Inflammatory Responses and Potential Uses in Vaccination in Cancer and Infectious Diseases. J Immunol Res 2015, 832057 (2015).

43. Guha-Niyogi, A., Sullivan, D. R. \& Turco, S. J. Glycoconjugate structures of parasitic protozoa. Glycobiology 11, 45R-59R (2001).

44. Camargo, M. M. et al. Glycosylphosphatidylinositol-anchored mucin-like glycoproteins isolated from Trypanosoma cruzi trypomastigotes initiate the synthesis of proinflammatory cytokines by macrophages. J Immunol 158, 5890-5901 (1997).

45. Charbeneau, R. P. \& Peters-Golden, M. Eicosanoids: mediators and therapeutic targets in fibrotic lung disease. Clin Sci 108, 479-491 (2005).

46. Dai, L. et al. Lipoteichoic acid (LTA) and lipopolysaccharides (LPS) from periodontal pathogenic bacteria facilitate oncogenic herpesvirus infection within primary oral cells. PLoS One 9, e101326 (2014).

47. Iliev, D. B., Hansen, T., Jørgensen, S. M., Krasnov, A. \& Jørgensen, J. B. CpG- and LPS-activated MAPK signaling in in vitro cultured salmon (Salmo salar) mononuclear phagocytes. Fish Shellfish Immunol 35, 1079-1085 (2013).

48. Elcombe, S. E. et al. Dectin-1 regulates IL-10 production via a MSK1/2 and CREB dependent pathway and promotes the induction of regulatory macrophage markers. PLoS One 8, e60086 (2013). 
49. Feng, G. J. et al. Extracellular signal-related kinase (ERK) and p38 mitogen-activated protein (MAP) kinases differentially regulate the lipopolysaccharide-mediated induction of inducible nitric oxide synthase and IL-12 in macrophages: Leishmania phosphoglycans subvert macr. IImmunol 163, 6403-6412 (1999).

50. Olekhnovitch, R. \& Bousso, P. Induction, Propagation, and Activity of Host Nitric Oxide: Lessons from Leishmania Infection. Trends Parasitol 31, 653-664 (2015).

51. Miranda, M. M. et al. Nitric oxide and Brazilian propolis combined accelerates tissue repair by modulating cell migration, cytokine production and collagen deposition in experimental leishmaniasis. PLoS One 10, e0125101 (2015).

52. Maspi, N., Abdoli, A. \& Ghaffarifar, F. Pro- and anti-inflammatory cytokines in cutaneous leishmaniasis: a review. Pathog Glob Heal. 110, 247-260 (2016)

53. Cunha, C. F. et al. Cytotoxic cell involvement in human cutaneous leishmaniasis: assessments in active disease, under therapy and after clinical cure. Parasite Immunol 38, 244-254 (2016).

54. Lima, M. H. F. et al. Leishmania infantum parasites subvert the host inflammatory response through the adenosine A2A receptor to promote the establishment of infection. Front. Immunol. 8, 1-12 (2017).

55. Odegaard, J. I. et al. Macrophage-specific PPARgamma controls alternative activation and improves insulin resistance. Nature 447, $1116-1120$ (2007).

56. Adapala, N. \& Chan, M. M. Long-term use of an antiinflammatory, curcumin, suppressed type 1 immunity and exacerbated visceral leishmaniasis in a chronic experimental model. Lab Invest 88, 1329-1339 (2008).

57. Araújo, C. V. et al. A ppar $\gamma$ agonist enhances bacterial clearance through neutrophil extracellular trap formation and improves survival in sepsis. Shock 45, 393-403 (2016).

58. Lakshmi, S. P., Reddy, A. T., Banno, A. \& Reddy, R. C. PPAR Agonists for the Prevention and Treatment of Lung Cancer. PPAR Res 2017, 8252796 (2017)

59. Fu, Y. et al. A novel pyrazole-containing indolizine derivative suppresses NF- $\kappa B$ activation and protects against TNBS-induced colitis via a PPAR- $\gamma$-dependent pathway. Biochem Pharmacol, doi:https://doi.org/10.1016/j.bcp.2017.03.013 (2017).

60. Zolezzi, J. M. et al. PPARs in the central nervous system: roles in neurodegeneration and neuroinflammation. Biol Rev Camb Philos Soc, doi:https://doi.org/10.1111/brv.12320 (2017).

61. Assunção, L. S. et al. Schistosomal-derived lysophosphatidylcholine triggers M2 polarization of macrophages through PPAR $\gamma$ dependent mechanisms. Biochim Biophys Acta 1862, 246-254 (2017).

62. Almeida, P. E. et al. Mycobacterium bovis bacillus Calmette-Guérin infection induces TLR2-dependent peroxisome proliferatoractivated receptor gamma expression and activation: functions in inflammation, lipid metabolism, and pathogenesis. J Immunol 183, 1337-1345 (2009).

63. Chan, M. M., Adapala, N. \& Chen, C. Peroxisome Proliferator-Activated Receptor- $\gamma$-Mediated Polarization of Macrophages in Leishmania Infection. PPAR Res 2012, 796235 (2012).

64. Luz, N. F. et al. Heme oxygenase-1 promotes the persistence of Leishmania chagasi infection. J Immunol 188, 4460-4467 (2012).

65. Soares, R. P. et al. Leishmania chagasi: lipophosphoglycan characterization and binding to the midgut of the sand fly vector Lutzomyia longipalpis. Mol Biochem Parasitol 121, 213-224 (2002).

66. Soares, R. P. et al. In Vitro Inhibition of Leishmania Attachment to Sandfly Midguts and LL-5 Cells by Divalent Metal Chelators, Anti-gp63 and Phosphoglycans. Protist 168, 326-334 (2017).

67. Araújo-Santos, T. et al. Lutzomyia longipalpis saliva triggers lipid body formation and prostaglandin $\mathrm{E}_{2}$ production in murine macrophages. PLoS Negl. Trop. Dis. 4, e873 (2010).

\section{Acknowledgements}

We thank Andrezza Souza for technical and logistic support. The authors would like to thank Andris K. Walter for English language revision and manuscript copyediting assistance. This work was supported by the Brazilian National Research Council (CNPq, 400135/2014-0 to VMB), Fundação de Amparo à Pesquisa do Estado da Bahia (FAPESB, INT0015/2016 to VMB and 305065/2016-5 to RPS), Fundação de Amparo à Pesquisa do Estado do Rio de Janeiro (FAPERJ, E-26/203684/2014 to PTB) and Fundação de Amparo à Pesquisa do Estado de Minas Gerais (FAPEMIG, PPM-00102-16 to RPS). VMB, RPS and PTB are senior investigators from CNPq. NFL and MLS are fellowship from CNPq. The funders had no role in study design, data collection and analysis, decision to publish, or preparation of the manuscript.

\section{Author Contributions}

T.A.S., J.B.L., P.T.B., R.P.S. and V.M.B. conceived and designed the study. T.A.S., J.B.L., M.L.S., A.B., N.F.L., F.H.J.S., S.M.P., P.F.E. and I.C.I. performed the experiments. T.A.S., J.B.L., M.L.S., A.B., A.D., P.T.B., R.P.S. and V.M.B. contributed with data analysis. P.T.B., R.P.S. and V.M.B. provided materials and infrastructural support. T.A.S., J.B.L., R.P.S., P.T.B. and V.M.B. wrote and revised the manuscript. All authors read and approved the final version of the manuscript. The authors declare that they do not have a commercial association that might pose a conflict of interest.

\section{Additional Information}

Supplementary information accompanies this paper at https://doi.org/10.1038/s41598-017-14229-8.

Competing Interests: The authors declare that they have no competing interests.

Publisher's note: Springer Nature remains neutral with regard to jurisdictional claims in published maps and institutional affiliations.

Open Access This article is licensed under a Creative Commons Attribution 4.0 International License, which permits use, sharing, adaptation, distribution and reproduction in any medium or format, as long as you give appropriate credit to the original author(s) and the source, provide a link to the Creative Commons license, and indicate if changes were made. The images or other third party material in this article are included in the article's Creative Commons license, unless indicated otherwise in a credit line to the material. If material is not included in the article's Creative Commons license and your intended use is not permitted by statutory regulation or exceeds the permitted use, you will need to obtain permission directly from the copyright holder. To view a copy of this license, visit http://creativecommons.org/licenses/by/4.0/.

(C) The Author(s) 2017 